American Journal of Applied Sciences 3 (1): 1682-1684, 2006

ISSN 1546-9239

(c) 2006 Science Publications

\title{
Ti: Cognitive Function in Diabetes Mellitus Patients
}

\author{
Rostam Seyfaddini \\ Department of Neurology, Shafa Medical Center, Kerman University of Medical Sciences, Iran
}

\begin{abstract}
Neuropathies are one of the most common complications in diabetic patients. Several studies determined relationship between cognitive disorders and diabetes mellitus (DM). The historical cohort study was designed to distinguish relation of DM and cognitive decrements. The Wisconsin Card Sort Test (WCST) and Mini-Mental Status Exam (MMSE) were conducted for evaluation of cognitive problems. Fifty diabetic patient and 48 control participants were included to study. Exposure and no-exposure groups had no significant difference regarding sex, age and educational level. Cognitive problems were 8 times more in diabetics than control group ( $\mathrm{RR}=8.2 \% 95 \mathrm{CI}=2.15$ - 31.4). Such as differences were in color, shape, and combined items between two groups. The MMSE results were not significant differences between both groups. The findings of our study strongly confirmed relationship between DM and cognitive decrements. These results have been shown in previous studies by different investigators.
\end{abstract}

Key words: Wisconsin card sort test, diabetes mellitus, cognitive problems, Iran

\section{INTRODUCTION}

Diabetic patients report wide range of neuropsychiatry problems. Diabetes mellitus (DM) has been known as a modifiable risk factor for early cognitive decline. Many investigations have examined diabetes in relation to early cognitive decline ${ }^{[1-11]}$. Some studies report impairments on tests relying on problem solving skills, whereas other studies report deficits in psychomotor efficiency, learning and memory, and visuospatial abilities ${ }^{[12-15]}$. This heterogeneity is probably caused by differences in patient characteristics and the psychometric paradigms used. Consequently, the exact pattern and magnitude of cognitive dysfunction is still unclear. Aging causes cortical brain atrophy that leads to loss of memory and cognitive impairment overaly. Age need to adjust for evaluating cognitive functions.

Other variable that effects on cognitive dysfunction and its severity is educational level in these subjects ${ }^{[14,16]}$. The aim of current study was to assess the associations between DM and cognitive function in middle-age subjects with controlling educational level.

\section{MATERIALS AND METHODS}

In a historical cohort study, 50 diabetic patients were selected randomly from known cases of diabetes mellitus in "Kerman diabetes center" as the group experiencing exposure. Patients were 25 to 65 years old and their disease had been diagnosed in the last 5 years. These patients were in different educational levels. As the group of no exposure, 50 patients coming to dermatology clinic who were matched according to sex, age, and educational level were selected.

All samples were assessed by a neurologist through neurological physical exams and were tested by blinded cognitive function tests. To assess cognitive function three methods were used:

* Frontal lobe cognitive test named "Wisconsin Card

Sort Test" (WCST) was first used.

This test was innovated by Grant \& Berg in 1948 to assess abstract reasoning. 64 cards were used .In each of these cards 1-4 identical shapes of triangle, star, circle or cross in the color of red, green, blue or yellow were printed. Cards were all different. Each participant was given a group of cards and was to put them in place regarding the concept that he gets from our model after some explanations (the same for all participants). A score of 1-6 was given to each participant and for each mistake one score was decreased. The test was classified in 7 types: 1-Color, 2-number, 3-shape, 4Color and shape, 5-Number and shape, 6-Color and number, 7-Color, number and shape. In each classification the participant had to understand what we meant. At the end, sum of the scores was considered as the total score.

* Three other lobes' function test, which was performed to rule out other lobes problems in specific conditions.

* Mini mental state exam (MMSE), which was done to assess psychological status of the participants.

After gathering the data, analysis was performed using SPSS software (version 9.5). Fisher's exact test and independent samples t-test were used to find differences. Relative risk with confidence interval of

Corresponding Author: $\quad$ Rostam Seyfaddini (MD), Department of Neurology, Shafa Medical Center. Kerman University of Medical Sciences, Kerman, Iran, Tel: +989131405232 
Am. J. Appl. Sci., 3 (1): 1682-1684, 2006

Table1: Distribution of diabetics and non-diabetics in different WCST classes

\begin{tabular}{|c|c|c|c|c|}
\hline Exposure/Disorder & Diabetic & Non-diabetic & $P$ value & Relative risk \\
\hline \multicolumn{5}{|l|}{ Disorder in color } \\
\hline Positive & 20 & 2 & & 2.3 \\
\hline Negative & 30 & 46 & $\mathrm{P}<0.0001$ & $(1.7-3.1)$ \\
\hline Disorder & & & & \\
\hline Positive & 9 & 6 & & 1.2 \\
\hline Negative & 41 & 42 & $\mathrm{P}=0.577$ & $(0.76-1.9)$ \\
\hline \multicolumn{5}{|l|}{ Disorder in shape } \\
\hline Positive & 32 & 2 & & 3.34 \\
\hline Negative & 18 & 46 & $\mathrm{P}<0.0001$ & $(2.24-5)$ \\
\hline Disorder in color and shape & & & & \\
\hline Positive & 35 & 5 & & 3.38 \\
\hline Negative & 15 & 43 & $\mathrm{P}<0.0001$ & $(2.15-5.3)$ \\
\hline \multicolumn{5}{|l|}{ Disorder in number and shape } \\
\hline Positive & 39 & 11 & & 3.4 \\
\hline Negative & 11 & 37 & $\mathrm{P}<0.0001$ & $(2-5.83)$ \\
\hline \multicolumn{5}{|l|}{ Disorder in color and number } \\
\hline Positive & 29 & 16 & & 1.6 \\
\hline Negative & 21 & 32 & $\mathrm{P}=0.016$ & $(1.1-2.4)$ \\
\hline \multicolumn{5}{|l|}{ Disorder in color, number and shape } \\
\hline Positive & 40 & 6 & & 4.5 \\
\hline Negative & 10 & 42 & $\mathrm{P}<0.001$ & $(2.56-8)$ \\
\hline
\end{tabular}

95\% was calculated. In all tests, the significance level of $5 \%$ was considered.

\section{RESULTS}

From one hundred participants who were included in the study, two of them were excluded according to no tendency for participation (from "No- exposure group"). This way "exposure group" (diabetics) and "no-exposure group" (non diabetics) included 50 and 48 participants respectively.

From 34 women who participated in the study, 16 were diabetic and the remainders were non-diabetic. Of 64 men, 34 were diabetic and 30 were Non-diabetic. Exposure and no-exposure groups had no significant difference regarding sex, age and educational level.

According to "Wisconsin card sort test" (WCST) results, diabetics and non-diabetics were different and cognitive problems were more frequently seen in diabetics rather than non-diabetic ones ( $R R=8.21$ $\% 95 \mathrm{CI}=2.15-31.4$ ) (Fig. 1). Such a difference was also found in different classes of WCST (Table 1). MMSE results showed no significant difference between groups, which means two groups of study were not different regarding psychological status (Table 2).

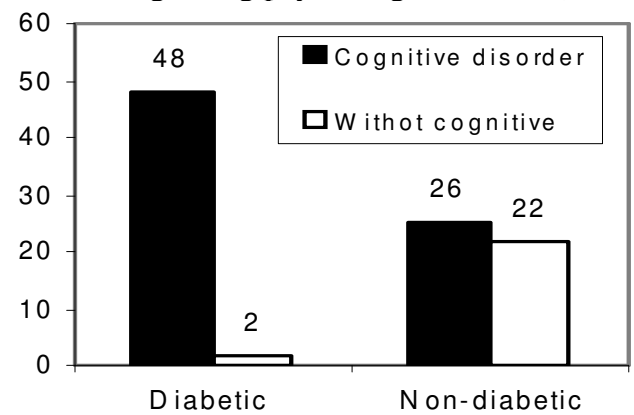

$\mathrm{RR}=8.21 \mathrm{CI} 95 \%=2.15-31.4$

Fig 1: Cognitive disorder frequency distribution among diabetics and non-diabetics
Table 2: Distribution of diabetics and non-diabetics by MMSE results

\begin{tabular}{lccc}
\hline Exposure/Disorder & Diabetic & Non-diabetic & Total \\
\hline Positive MMSE & 6 & 3 & 9 \\
Negative MMSE & 44 & 45 & 89 \\
Total & 50 & 48 & 98 \\
\hline
\end{tabular}

Fisher's exact test; $\quad \mathrm{P}=0.264$

\section{DISCUSSION}

The findings of our study strongly support the hypothesis that there is a relationship between cognitive dysfunction and diabetes mellitus. Diabetics were involved cognitive impairments 8 times more than nondiabetic subjects. Severity of impairment was mild to moderate in this study. Overall, summarizing all studies and all cognitive domains (overall cognition), there is a modest but highly significant difference in cognitive performance between diabetic patients and non-diabetic control subjects. The pattern of cognitive findings does not suggest an overall impairment of cognitive function but is characterized by a slowing of mental speed and a diminished mental flexibility ${ }^{[17]}$. Although the magnitude of most of these cognitive problems is relatively modest moderate forms of cognitive dysfunction can potentially hamper everyday activities, and they can be expected to present problems in more demanding situations ${ }^{[17]}$.

As such, Sinclair et al determined a negative impact on the quality of life of diabetic patients with cognitive dysfunction ${ }^{[18]}$.

A major strength of our study was matching of case and control as age and educational level. We examined patients with face-to-face interview by WCST and MMSE that are standardized for Iranian people and apply for cognitive impairment. It seems we had a good estimation of cognitive problems in individuals. Logroscino and co-workers demonstrated significant difference between diabetic and non-diabetic elderly 
female subjects in cognitive decrements. They applied phone interview for determining of cognitive decrements and they have pointed out that this method maybe underestimate the diagnosis of cognitive problems.

Ryan and Geckle examined at least 16 test of cognitive function assessment and loaded 4 main component for cognitive evaluation; Learning, memory of stories, problem solving, and psychomotor efficiency. They notified that direct interview to patients is more reliable method for assessing of cognitive decrement ${ }^{[19]}$.

Diabetes is a systemic disease that affects multiple organs and cause dysfunction of them. Neurological impacts of diabetes have been established. Reviewing of investigations has cleared cognitive decrements in diabetic subjects, definitely. Our study confirmed this relationship. However the result of MMSE was not difference in diabetic and non-diabetic subjects. There were some limitations in our study. First, we examined subject once thus they needed several assessment for exact confirmation of cognitive decrements. Second, treatment and medication of diabetes was not registered in this study. In addition, because of historical study we could not find the duration and onset of disorders in patients. We recommend further investment about this course.

\section{REFERENCES}

1. Gregg, E.W., K. Yaffe, J.A. Cauley, D.B. Rolka, T.L. Blackwell and K.M. Narayan et al., 2000. Is diabetes associated with cognitive impairment and cognitive decline among older women? Study of Osteoporotic Fractures Research Group. Arch. Intern. Med., 160: 174-80.

2. Knopman, D., L.L. Boland, T. Mosley, G. Howard, D. Liao and M. Szklo et al., 2001. Cardiovascular risk factors and cognitive decline in middle-aged adults. Neurology, 56: 42-8.

3. Kalmijn, S., E.J. Feskens, L.J. Launer, T. Stijnen, and D. Kromhout, 1995. Glucose intolerance, hyperinsulinaemia and cognitive function in a general population of elderly men. Diabetologia, 38: 1096-102.

4. Scott, R.D., D. Kritz-Silverstein, E. Barrett-Connor and W.C. Wiederholt, 1998. The association of non-insulin-dependent diabetes mellitus and cognitive function in an older cohort. J. Am. Geriatr. Soc., 46: 1217-22.

5. Fontbonne, A., C. Berr, P. Ducimetiere and A. Alperovitch, 2001. Changes in cognitive abilities over a 4-year period are unfavorably affected in elderly diabetic subjects: results of the epidemiology of vascular aging study. Diabetes Care, 24: 366-70.

6. Stolk, R.P., M.M. Breteler, A. Ott, H.A. Pols, S.W. Lamberts and D.E. Grobbee et al., 1997. Insulin and cognitive function in an elderly population. The Rotterdam study. Diabetes Care, 20: 792-5.
7. Elias, P.K., M.F. Elias, R.B. D’Agostino, L.A. Cupples, P.W. Wilson and H. Silbershatz et al., 1997. NIDDM and blood pressure as risk factors for poor cognitive performance. The Framingham study. Diabetes Care, 20: 1388-95.

8. Rodriguez-Saldana, J., J.E. Morley, M.T. Reynoso, C.A. Medina, P. Salazar and E. Cruz et al., 2002. Diabetes mellitus in a subgroup of older Mexicans: prevalence, association with cardiovascular risk factors, functional and cognitive impairment, and mortality. J. Am. Geriatr. Soc., 50: 111-6.

9. Nguyen, H.T., S.A. Black, L.A. Ray, D.V. Espino and K.S. Markides, 2002. Predictors of decline in MMSE scores among older Mexican Americans. J. Gerontol. A Biol. Sci. Med. Sci., 57: M181-5.

10. Wu, J.H., M.N. Haan, J. Liang, D. Ghosh, H.M. Gonzalez and W.H. Herman, 2003. Impact of diabetes on cognitive function among older Latinos: a population-based cohort study. J. Clin. Epidemiol., 56: 686-93.

11. Vanhanen, M., J. Kuusisto, K. Koivisto, L. Mykkanen, E.L. Helkala and T. Hanninen et al., 1999. Type-2 diabetes and cognitive function in a non-demented population. Acta Neurol. Scand., 100: 97-101.

12. Deary, I.J., J.R. Crawford, D.A. Hepburn, S.J. Langan, L.M. Blackmore and B.M. Frier, 1993. Severe hypoglycemia and intelligence in adult patients with insulin-treated diabetes. Diabetes, 42: 341-344.

13. Ryan, C.M., T.M. Williams, T.J. Orchard and D.N. Finegold, 1992. Psychomotor slowing is associated with distal symmetrical polyneuropathy in adults with diabetes mellitus. Diabetes, 41: 107-113.

14. Sachon, C., A. Grimaldi, J.P. Digy, B. Pillon, B. Dubois and F. Thervet, 1992. Cognitive function, insulin-dependent diabetes and hypoglycemia. J. Intern. Med., 231: 471-475.

15. Wredling, R., S. Levander, U. Adamson and P.E. Lins, 1990. Permanent neuropsychological impairment after recurrent episodes of severe hypoglycaemia in man. Diabetologia, 33: 152-157.

16. Hershey, T., S. Craft, N. Bhargava and N.H. White, 1997. Memory and insulin dependent diabetes mellitus (IDDM): effects of childhood onset and severe hypoglycemia. J. Int. Neuropsychol. Soc., 3: 509-520.

17. Brands, A.M., G.J., E.H., L.J. and R.P., 2005. The effects of type 1 diabetes on cognitive performance: a meta-analysis. Diabetes Care, 28: 726-35.

18. Sinclair, A.J., A.J. Girling and A. Bayer, 2000. Cognitive dysfunction in older subjects with diabetes mellitus: impact on diabetes selfmanagement and use of care services. Diabetes Res. Clin. Pract., 50: 203-212.

19. Ryan, C.M. and M.O. Geckle, 2000. Circumscribed cognitive dysfunction in middle-aged adults with type 2 diabetes. Diabetes Care, 23: 1486-93. 